\title{
Short-term at-the-money asymptotics under stochastic volatility models
}

\author{
Omar El Euch \\ École Polytechnique \\ Masaaki Fukasawa \\ Graduate School of Engineering Science, Osaka University \\ 1-3 Machikaneyama, Toyonaka, Osaka, JAPAN \\ fukasawa@sigmath.es.osaka-u.ac.jp \\ Jim Gatheral \\ Baruch College, The City University of New York \\ Mathieu Rosenbaum \\ École Polytechnique
}

22 March 2019

\begin{abstract}
A small-time Edgeworth expansion of the density of an asset price is given under a general stochastic volatility model, from which asymptotic expansions of put option prices and at-the-money implied volatilities follow. A limit theorem for at-the-money implied volatility skew and curvature is also given as a corollary. The rough Bergomi model is treated as an example.
\end{abstract}

\section{Introduction}

Stochastic volatility models are extensions of the Black-Scholes model that explain a number of empirical evidences. The Heston and SABR models among them are popular in financial practice owing to (semi-)analytic (approximation) formulas for the vanilla option prices or the option-implied volatilities. For a practical guide on stochastic volatility modeling, we refer to [12]. Recently, attracting much attention is a class of stochastic volatility models where the volatility is driven by a fractional Brownian motion with the Hurst parameter smaller than $1 / 2$. This is due to their consistency to a power law for the term 
structure of the implied volatility skew that has been empirically recognized; see [1, 3, 6, 8, 10, 11, 13, 14]. The small Hurst parameter implies in particular that the volatility path is rougher than a Brownian motion and so, this class is often referred as the rough volatility models. Since the models do not admit of explicit expressions for option prices or implied volatilities, the above mentioned consistency has been discussed through asymptotic analyses.

The aim of this paper is to provide a general framework under which the short-term asymptotics of the at-the-money implied volatility is studied. Here, by short-term asymptotics, we mean the asymptotics with time-to-maturity $\theta \rightarrow 0$. By at-the-money, we mean a regime of log-moneyness $k=O(\sqrt{\theta})$. The framework is for a general continuous stochastic volatility model. The rough Bergomi model introduced by [3] is treated as an example. The asymptotic expansion of the at-the-money implied volatility is given up to the secondorder.

The first-order expansion was already given in [10] by a different method. For the SABR model, Osajima [19] gave the second-order expansion based on the Watanabe-Yoshida theory; see e.g., [15, 22]. For a Markov stochastic volatility model with jumps, Medvedev and Scaillet [16, 17] derived the expansion by a formal computation. For the Markov diffusion case, Pagliarani and Pascucci [20] proved the validity of the Taylor expansion. An expansion of the at-the-money implied volatility skew is derived under a Lévy jump model with Markov stochastic volatility by Figueroa-López and Ólafsson [5]. Beside these results for the at-the-money regime, considering near-the-money, that is, a moderate deviation regime, Friz et al. [7] derived the asymptotic skew and curvature of the implied volatility by assuming the asymptotic behavior of the density function of the underlying asset price. Recently, Bayer et al. [4] has extended the moderate deviation analysis to a rough volatility model.

In this paper, we introduce a novel approach based on the conditional Gaussianity of a continuous stochastic volatility model to prove the validity of a second order density expansion, from which follow expansions of the option prices and the implied volatility as well as the asymptotic skew and curvature formula. In contrast to [15, 19, 22], we do not rely on the Malliavin calculus, which enables us to treat effectively the rough volatility models. In contrast to the elementary method of [10], our approach can be extended to higher-order expansions without any additional theoretical difficulty. We choose the square root of the forward variance, that is, the fair strike of a variance swap, as the leading term of our asymptotic expansion, while a recent work [2] studies the difference between the implied volatility and the fair strike of a volatility swap in terms of the Malliavin derivatives.

The paper is organized as follows. In Section 2, we describe assumptions and general results. In Section 3, we give the proofs of the general results. In Section 4, we treat regular stochastic volatility models. In Section 5, we show that the rough Bergomi model fits into the framework as well and compute the coefficients of the expansion for this particular model. 


\section{Framework}

\subsection{Assumptions}

Let $(\Omega, \mathcal{F}, Q)$ be a probability space equipped with a filtration $\left\{\mathcal{F}_{t} ; t \geq 0\right\}$ satisfying the usual assumptions. A $\log$ price process $Z$ is assumed to follow

$$
\mathrm{d} Z_{t}=r \mathrm{~d} t-\frac{1}{2} v_{t} \mathrm{~d} t+\sqrt{v_{t}} \mathrm{~d} B_{t}
$$

where $r \in \mathbb{R}$ stands for an interest rate and $v$ is a positive continuous process adapted to a smaller filtration $\left\{\mathcal{G}_{t} ; t \geq 0\right\}$, of which the square root is called the volatility of $Z$. The Brownian motion $B$ is decomposed as

$$
\mathrm{d} B_{t}=\rho_{t} \mathrm{~d} W_{t}+\sqrt{1-\rho_{t}^{2}} \mathrm{~d} W_{t}^{\prime},
$$

where $W^{\prime}$ is an $\left\{\mathcal{F}_{t}\right\}$-Brownian motion independent of $\mathcal{G}_{t}$ for all $t \geq 0, W$ is a $\left\{\mathcal{G}_{t}\right\}$-Brownian motion and $\rho$ is a progressively measurable processes with respect to $\left\{\mathcal{G}_{t}\right\}$ and taking values in $[-1,1]$. A typical situation for stochastic volatility models, including the Heston, SABR and rough Bergomi models, is that $\left(W, W^{\prime}\right)$ is a two dimensional $\left\{\mathcal{F}_{t}\right\}$-Brownian motion and $\left\{\mathcal{G}_{t}\right\}$ is the filtration generated by $W$, that is,

$$
\mathcal{G}_{t}=\mathcal{N} \vee \sigma\left(W_{s} ; s \leq t\right),
$$

where $\mathcal{N}$ is the null sets of $\mathcal{F}$. Denote by $\|\cdot\|_{p}$ the $L^{p}$ norm under $Q$. Our key assumption is the following: for any $p>0$,

$$
\sup _{\theta \in(0,1)}\left\|\frac{1}{\theta} \int_{0}^{\theta} v_{t} \mathrm{~d} t\right\|_{p}<\infty, \sup _{\theta \in(0,1)}\left\|\left\{\frac{1}{\theta} \int_{0}^{\theta} v_{t}\left(1-\rho_{t}^{2}\right) \mathrm{d} t\right\}^{-1}\right\|_{p}<\infty .
$$

This is satisfied by standard stochastic volatility models (with correlation parameter $|\rho|<1$ ) but not by local volatility models that correspond to $\rho \equiv 1$.

An arbitrage-free price $p(K, \theta)$ of a put option at time 0 with strike $K>0$ and maturity $\theta>0$ is given by

$$
p(K, \theta)=e^{-r \theta} E\left[\left(K-\exp \left(Z_{\theta}\right)\right)_{+}\right]=e^{-r \theta} \int_{0}^{K} Q\left(\log x \geq Z_{\theta}\right) \mathrm{d} x .
$$

The forward variance curve $v_{0}(t)$ at time 0 is defined by $v_{0}(t)=E\left[v_{t}\right]$. Changing variable as

$$
x=F \exp \left(\zeta \sigma_{0}(\theta)\right), \quad F=\exp \left(r \theta+Z_{0}\right),
$$

where

$$
\sigma_{0}(\theta)=\sqrt{\int_{0}^{\theta} v_{0}(t) \mathrm{d} t}
$$


we have

$$
\frac{p\left(F e^{z \sigma_{0}(\theta)}, \theta\right)}{F \sigma_{0}(\theta)}=e^{-r \theta} \int_{-\infty}^{z} Q\left(\zeta \geq X_{\theta}\right) e^{\sigma_{0}(\theta) \zeta} \mathrm{d} \zeta
$$

where

$$
X_{\theta}=-\frac{1}{2 \sigma_{0}(\theta)}\langle M\rangle_{\theta}+\frac{1}{\sigma_{0}(\theta)} M_{\theta}, M_{\theta}=\int_{0}^{\theta} \sqrt{v_{t}} \mathrm{~d} B_{t},\langle M\rangle_{\theta}=\int_{0}^{\theta} v_{t} \mathrm{~d} t .
$$

Based on this expression, the asymptotic behavior of put option prices is studied through the asymptotic distribution of $X_{\theta}$. From the martingale central limit theorem 11 it is not difficult to see that $X_{\theta}$ converges in law to the standard normal distribution as $\theta \rightarrow 0$. To determine higher-order asymptotic distribution, we assume the following structure: there exists a family of random vectors

$$
\left\{\left(M_{\theta}^{(0)}, M_{\theta}^{(1)}, M_{\theta}^{(2)}, M_{\theta}^{(3)}\right) ; \theta \in(0,1)\right\}
$$

such that

1. the law of $M_{\theta}^{(0)}$ is standard normal for all $\theta>0$,

2.

$$
\sup _{\theta \in(0,1)}\left\|M_{\theta}^{(i)}\right\|_{p}<\infty, \quad i=1,2,3
$$

for all $p>0$ and

3. for some $H \in(0,1 / 2]$ and $\epsilon \in(0, H)$,

$$
\begin{aligned}
& \lim _{\theta \rightarrow 0} \theta^{-2 H-2 \epsilon}\left\|\frac{M_{\theta}}{\sigma_{0}(\theta)}-M_{\theta}^{(0)}-\theta^{H} M_{\theta}^{(1)}-\theta^{2 H} M_{\theta}^{(2)}\right\|_{1+\epsilon}=0, \\
& \lim _{\theta \rightarrow 0} \theta^{-H-2 \epsilon}\left\|\frac{\langle M\rangle_{\theta}}{\sigma_{0}(\theta)^{2}}-1-\theta^{H} M_{\theta}^{(3)}\right\|_{1+\epsilon}=0 .
\end{aligned}
$$

Further, we assume the existence of the derivatives

$$
\begin{aligned}
& a_{\theta}^{(i)}(x)=\frac{\mathrm{d}}{\mathrm{d} x}\left\{E\left[M_{\theta}^{(i)} \mid M_{\theta}^{(0)}=x\right] \phi(x)\right\}, \quad i=1,2,3, \\
& b_{\theta}(x)=\frac{\mathrm{d}^{2}}{\mathrm{~d} x^{2}}\left\{E\left[M_{\theta}^{(1)} \mid M_{\theta}^{(0)}=x\right] \phi(x)\right\} \\
& c_{\theta}(x)=\frac{\mathrm{d}^{2}}{\mathrm{~d} x^{2}}\left\{E\left[\left|M_{\theta}^{(1)}\right|^{2} \mid M_{\theta}^{(0)}=x\right] \phi(x)\right\}
\end{aligned}
$$

in the Schwartz space (i.e., the space of the rapidly decreasing smooth functions), where $\phi$ is the standard normal density.

\footnotetext{
1 The martingale central limit theorem for one-dimensional continuous local martingales is proved as follows. Let $M^{n}$ be a continuous local martingale with $\left\langle M^{n}\right\rangle_{1} \rightarrow 1$ in probability. By the Dambis-Dubins-Schwarz theorem, $M^{n}=W_{\left\langle M^{n}\right\rangle}^{n}$ for a Brownian motion $W^{n}$. Since $\left(W^{n},\left\langle M^{n}\right\rangle_{1}\right) \rightarrow$ $(W, 1)$ in law, by the continuous mapping theorem, we conclude $M_{1}^{n} \rightarrow W_{1}$ in law.
} 
As will be discussed in Section 4, regular stochastic volatility models satisfy these assumptions with $H=1 / 2$, where (3) is a consequence of the Itô-Taylor expansion. In Section 5, we see that the rough Bergomi model, where the volatility is driven by a fractional Brownian motion, satisfies these assumptions with $H$ being the Hurst parameter of the fractional Brownian motion.

\subsection{General results}

The fundamental result in this paper is the following.

Theorem 2.1 The law of $X_{\theta}$ admits a density $p_{\theta}$, and for any $\alpha \in \mathbb{N} \cup\{0\}$,

$$
\sup _{x \in \mathbb{R}}\left(1+x^{2}\right)^{\alpha}\left|p_{\theta}(x)-q_{\theta}(x)\right|=o\left(\theta^{2 H}\right)
$$

as $\theta \rightarrow 0$, where

$$
\begin{aligned}
q_{\theta}(x)= & \phi\left(x+\frac{\sigma_{0}(\theta)}{2}\right)-\theta^{H}\left(a_{\theta}^{(1)}\left(x+\frac{\sigma_{0}(\theta)}{2}\right)-\frac{\sigma_{0}(\theta)}{2} a_{\theta}^{(3)}\left(x+\frac{\sigma_{0}(\theta)}{2}\right)\right) \\
& -\theta^{2 H}\left(a_{\theta}^{(2)}(x)-\frac{1}{2} c_{\theta}(x)\right) .
\end{aligned}
$$

The proof is given in Section 3.2. In order to derive a neat asymptotic expansion of the put option prices, we introduce an additional assumption which is satisfied by the models in Sections 4 and 5 .

Theorem 2.2 Suppose we have (5) with $q_{\theta}$ of the form

$$
\begin{aligned}
q_{\theta}(x)= & \phi\left(x+\frac{\sigma_{0}(\theta)}{2}\right)\left\{1+\kappa_{3}(\theta)\left(H_{3}\left(x+\frac{\sigma_{0}(\theta)}{2}\right)-\sigma_{0}(\theta) H_{2}\left(x+\frac{\sigma_{0}(\theta)}{2}\right)\right) \theta^{H}\right\} \\
& +\phi(x)\left(\kappa_{4}(\theta) H_{4}(x)+\frac{\kappa_{3}(\theta)^{2}}{2} H_{6}(x)\right) \theta^{2 H}
\end{aligned}
$$

with bounded functions $\kappa_{3}(\theta)$ and $\kappa_{4}(\theta)$ of $\theta$, where $H_{k}$ is the $k$ th Hermite polynomial:

$$
H_{1}(x)=x, H_{2}(x)=x^{2}-1, H_{3}(x)=x^{3}-3 x, H_{4}(x)=x^{4}-6 x^{2}+3, \ldots
$$

Then, for any $z_{0} \in \mathbb{R}$,

$$
\begin{aligned}
\frac{p\left(F e^{\sigma_{0}(\theta) z}, \theta\right)}{F e^{-r \theta} \sigma_{0}(\theta)}= & \frac{1}{\sigma_{0}(\theta)}\left(\Phi\left(z+\frac{\sigma_{0}(\theta)}{2}\right) e^{\sigma_{0}(\theta) z}-\Phi\left(z-\frac{\sigma_{0}(\theta)}{2}\right)\right) \\
& +\kappa_{3}(\theta) \phi\left(z+\frac{\sigma_{0}(\theta)}{2}\right) H_{1}\left(z+\frac{\sigma_{0}(\theta)}{2}\right) e^{\sigma_{0}(\theta) z} \theta^{H} \\
& +\phi(z)\left(\kappa_{4}(\theta) H_{2}(z)+\frac{\kappa_{3}(\theta)^{2}}{2} H_{4}(z)\right) \theta^{2 H}+o\left(\theta^{2 H}\right)
\end{aligned}
$$

uniformly in $z \leq z_{0}$. 
The proof is given in Section 3.3. Under the same assumption, an asymptotic expansion of the Black-Scholes implied volatility follows. Denote by $p_{\mathrm{BS}}(K, \theta, \sigma)$ the put option price with strike price $K$ and maturity $\theta$ under the Black-Scholes model with volatility parameter $\sigma>0$. Given a put option price $p(K, \theta), K=F e^{k}$, the Black-Scholes implied volatility $\sigma_{\mathrm{BS}}(k, \theta)$ is defined through

$$
p_{\mathrm{BS}}\left(K, \theta, \sigma_{\mathrm{BS}}(k, \theta)\right)=p(K, \theta) .
$$

The at-the-money implied volatility skew and curvature are defined respectively as the first and the second derivatives in $k$ of the Black-Scholes implied volatility at $k=0$. The skew behavior is especially important in order to argue the consistency of a model to the empirically observed power law.

Theorem 2.3 Suppose we have (5) with $q_{\theta}$ of the form (7). Then, for any $z \in \mathbb{R}$,

$$
\begin{aligned}
& \sigma_{\mathrm{BS}}(\sqrt{\theta} z, \theta) \\
& =\kappa_{2}\left\{1+\kappa_{3}\left(\frac{z}{\kappa_{2}}+\frac{\kappa_{2} \sqrt{\theta}}{2}\right) \theta^{H}+\left(\frac{3 \kappa_{3}^{2}}{2}-\kappa_{4}+\left(\kappa_{4}-3 \kappa_{3}^{2}\right) \frac{z^{2}}{\kappa_{2}^{2}}\right) \theta^{2 H}\right\}+o\left(\theta^{2 H}\right),
\end{aligned}
$$

where $\kappa_{2}=\kappa_{2}(\theta)=\sigma_{0}(\theta) / \sqrt{\theta}, \kappa_{3}=\kappa_{3}(\theta)$ and $\kappa_{4}=\kappa_{4}(\theta)$.

Theorem 2.4 Suppose we have (5) with $q_{\theta}$ of the form (7). Then,

$$
\begin{aligned}
& \partial_{k} \sigma_{\mathrm{BS}}(0, \theta)=\kappa_{3}(\theta) \theta^{H-1 / 2}+o\left(\theta^{2 H-1 / 2}\right), \\
& \partial_{k}^{2} \sigma_{\mathrm{BS}}(0, \theta)=2 \frac{\kappa_{4}(\theta)-3 \kappa_{3}(\theta)^{2}}{\kappa_{2}(\theta)} \theta^{2 H-1}+o\left(\theta^{2 H-1}\right) .
\end{aligned}
$$

The proofs are given in Sections 3.4 and 3.5 respectively.

Remark: The above asymptotic estimates are not uniform in $H$; the assumed stochastic expansion (3) is not uniform in $H$ and so, there seems no hope to have uniformity. We would have uniformity in $H \in\left[H_{0}, 1 / 2\right]$ for some $H_{0}>0$ if we could strengthen the condition (3) to uniform convergence on $\left[H_{0}, 1 / 2\right]$. It seems impossible to argue the uniformity in $H \in(0,1 / 2]$ because Lemma 3.2 below requires some $\epsilon-\delta$ argument depending on $H$.

\section{Proofs}

\subsection{Characteristic function expansion}

Here we give an asymptotic expansion of the characteristic function of $X_{\theta}$. Let

$$
Y_{\theta}=M_{\theta}^{(0)}+\theta^{H} M_{\theta}^{(1)}+\theta^{2 H} M_{\theta}^{(2)}-\frac{\sigma_{0}(\theta)}{2}\left(1+\theta^{H} M_{\theta}^{(3)}\right) .
$$


Lemma 3.1 Let $H \in(0,1 / 2]$ and $\epsilon \in(0, H)$ be constants under which (3) holds. Then, for any $\alpha \in \mathbb{N} \cup\{0\}$,

$$
\sup _{|u| \leq \theta^{-\epsilon}}\left|E\left[X_{\theta}^{\alpha} e^{i u X_{\theta}}\right]-E\left[Y_{\theta}^{\alpha} e^{i u Y_{\theta}}\right]\right|=o\left(\theta^{2 H+\varepsilon}\right) .
$$

Proof: Since $\left|e^{i x}-1\right| \leq|x|$, we have

$$
\begin{aligned}
\left|E\left[X_{\theta}^{\alpha} e^{i u X_{\theta}}\right]-E\left[Y_{\theta}^{\alpha} e^{i u Y_{\theta}}\right]\right| & \leq\left|E\left[\left(X_{\theta}^{\alpha}-Y_{\theta}^{\alpha}\right) e^{i u X_{\theta}}\right]\right|+\left|E\left[Y_{\theta}^{\alpha} e^{i u Y_{\theta}}\left(e^{i u\left(X_{\theta}-Y_{\theta}\right)}-1\right)\right]\right| \\
& \leq E\left[\left|X_{\theta}^{\alpha}-Y_{\theta}^{\alpha}\right|\right]+u E\left[\left|Y_{\theta}\right|^{\alpha}\left|X_{\theta}-Y_{\theta}\right|\right]
\end{aligned}
$$

By (1) and (2) respectively, $X_{\theta}$ and $Y_{\theta}$ have moments of any order. Therefore by the Hölder inequality,

$$
E\left[\left|Y_{\theta}\right|^{\alpha}\left|X_{\theta}-Y_{\theta}\right|\right] \leq C_{1}(\alpha, \epsilon)\left\|X_{\theta}-Y_{\theta}\right\|_{1+\epsilon}
$$

for a constant $C_{1}(\alpha, \epsilon)>0$. Since $X_{\theta}^{\alpha}-Y_{\theta}^{\alpha}=\left(X_{\theta}-Y_{\theta}\right) \sum_{\beta=0}^{\alpha-1}(-1)^{\beta} X_{\theta}^{\alpha-1-\beta} Y_{\theta}^{\beta}$, the Hölder inequality gives also

$$
E\left[\left|X_{\theta}^{\alpha}-Y_{\theta}^{\alpha}\right|\right] \leq C_{2}(\alpha, \epsilon)\left\|X_{\theta}-Y_{\theta}\right\|_{1+\epsilon}
$$

for a constant $C_{2}(\alpha, \epsilon)>0$. Since $\sigma_{0}(\theta)=O\left(\theta^{1 / 2}\right)$, we have $\left\|X_{\theta}-Y_{\theta}\right\|_{1+\epsilon}=$ $o\left(\theta^{2 H+2 \epsilon}\right)$ by (3), from which the result follows.

Lemma 3.2 Let $H$ and $\epsilon$ be as in Lemme 3.1 Then, for any $\delta \in[0,(H-\epsilon) / 3)$,

$$
\sup _{|u| \leq \theta^{-\delta}}\left|E\left[Y_{\theta}^{\alpha} e^{i u Y_{\theta}}\right]-E\left[e^{i u M_{\theta}^{(0)}}\left(\left(M_{\theta}^{(0)}\right)^{\alpha}+A\left(\alpha, u, M_{\theta}^{(0)}\right)+B\left(\alpha, u, M_{\theta}^{(0)}\right)\right)\right]\right|=o\left(\theta^{2 H+\epsilon}\right),
$$

where

$$
\begin{aligned}
A_{\theta}(\alpha, u, x)= & \left(i u x^{\alpha}+\alpha x^{\alpha-1}\right)\left(E\left[Y_{\theta} \mid M_{\theta}^{(0)}=x\right]-x\right), \\
B_{\theta}(\alpha, u, x)= & \left(-\frac{u^{2}}{2} x^{\alpha}+i u \alpha x^{\alpha-1}+\frac{\alpha(\alpha-1)}{2} x^{\alpha-2}\right) \\
& \times\left(\theta^{2 H} E\left[\left|M_{\theta}^{(1)}\right|^{2} \mid M_{\theta}^{(0)}=x\right]-\sigma_{0}(\theta) \theta^{H} E\left[M_{\theta}^{(1)} \mid M_{\theta}^{(0)}=x\right]+\frac{\sigma_{0}(\theta)^{2}}{4}\right) .
\end{aligned}
$$

Proof: This follows from the fact that

$$
\left|e^{i x}-1-i x+\frac{x^{2}}{2}\right| \leq \frac{|x|^{3}}{6}
$$

for all $x \in \mathbb{R}$. Indeed, this implies that

$$
\sup _{|u| \leq \theta^{-\delta}}\left|E\left[Y_{\theta}^{\alpha} e^{i u Y_{\theta}}\right]-E\left[Y_{\theta}^{\alpha} e^{i u M_{\theta}^{(0)}}\left(1+i u\left(Y_{\theta}-M_{\theta}^{(0)}\right)-\frac{u^{2}}{2}\left(Y_{\theta}-M_{\theta}^{(0)}\right)^{2}\right)\right]\right|=o\left(\theta^{2 H+\varepsilon}\right) \text {. }
$$

Expand $Y_{\theta}^{\alpha}=\left(M_{\theta}^{(0)}\right)^{\alpha}+\alpha\left(M_{\theta}^{(0)}\right)^{\alpha-1}\left(Y_{\theta}-M_{\theta}^{(0)}\right)+\ldots$ and take the conditional expectation given $M_{\theta}^{(0)}$ to obtain the result. 
Lemma 3.3 Define $\bar{q}_{\theta}(x)$ by

$$
\begin{aligned}
\bar{q}_{\theta}(x)= & \phi(x)-\theta^{H} a_{\theta}^{(1)}(x)-\theta^{2 H} a_{\theta}^{(2)}(x)-\frac{\sigma_{0}(\theta)}{2}\left(x \phi(x)-\theta^{H} a_{\theta}^{(3)}(x)\right) \\
& +\frac{\theta^{2 H}}{2} c_{\theta}(x)-\frac{\theta^{H} \sigma_{0}(\theta)}{2} b_{\theta}(x)+\frac{\sigma_{0}(\theta)^{2}}{8}\left(x^{2}-1\right) \phi(x),
\end{aligned}
$$

where $a_{\theta}^{(i)}, b_{\theta}$ and $c_{\theta}$ are defined by (4). Then,

$$
\int_{\mathbb{R}} e^{i u x} x^{\alpha} \bar{q}_{\theta}(x) \mathrm{d} x=E\left[e^{i u M_{\theta}^{(0)}}\left(\left(M_{\theta}^{(0)}\right)^{\alpha}+A\left(\alpha, u, M_{\theta}^{(0)}\right)+B\left(\alpha, u, M_{\theta}^{(0)}\right)\right)\right] .
$$

Proof: Since the density of $M_{\theta}^{(0)}$ is $\phi$ by the assumption, this simply follows from integration by parts.

\subsection{Density expansion}

Here we derive an asymptotic expansion of the density of $X_{\theta}$.

Lemma 3.4 There exists a density of $X_{\theta}$ and for any $\alpha, j \in \mathbb{N} \cup\{0\}$,

$$
\sup _{\theta \in(0,1)} \int|u|^{j}\left|E\left[X_{\theta}^{\alpha} e^{i u X_{\theta}}\right]\right| \mathrm{d} u<\infty
$$

Proof: Note that the distribution of $X_{\theta}$ is Gaussian conditionally on $\mathcal{G}_{\theta}$, with conditional mean

$$
U_{\theta}:=-\frac{1}{2 \sigma_{0}(\theta)}\langle M\rangle_{\theta}+\frac{1}{\sigma_{0}(\theta)} \int_{0}^{\theta} \sqrt{v_{t}} \rho_{t} \mathrm{~d} W_{t}
$$

and conditional variance

$$
V_{\theta}:=\frac{1}{\sigma_{0}(\theta)^{2}} \int_{0}^{\theta} v_{t}\left(1-\rho_{t}^{2}\right) \mathrm{d} t
$$

Therefore, for any bounded continuous function $f$, we have

$$
E\left[f\left(X_{\theta}\right)\right]=E\left[E\left[f\left(X_{\theta}\right) \mid \mathcal{G}_{\theta}\right]\right]=E\left[\int f(x) \phi\left(x, U_{\theta}, V_{\theta}\right) \mathrm{d} x\right],
$$

where $\phi(\cdot, u, v)$ is the density of the normal distribution with mean $u$ and variance $v$. This means that $X_{\theta}$ admits a density

$$
p_{\theta}(x)=E\left[\phi\left(x, U_{\theta}, V_{\theta}\right)\right]
$$

Furthermore, the density function is in the Schwartz space $\mathcal{S}$ and each Schwartz semi-norm is uniformly bounded in $\theta$ by (1). Therefore,

$$
\begin{aligned}
\sup _{\theta \in(0,1)} \int|u|^{j}\left|E\left[X_{\theta}^{\alpha} e^{i u X_{\theta}}\right]\right| \mathrm{d} u & =\sup _{\theta \in(0,1)} \int\left|\int u^{j} x^{\alpha} e^{i u x} p_{\theta}(x) \mathrm{d} x\right| \mathrm{d} u \\
& =\sup _{\theta \in(0,1)} \int\left|\int e^{i u x} \partial_{x}^{j}\left(x^{\alpha} p_{\theta}(x)\right) \mathrm{d} x\right| \mathrm{d} u<\infty
\end{aligned}
$$


since the Fourier transform is a continuous linear mapping from $\mathcal{S}$ to $\mathcal{S}$. I///

Proof of Theorem 2.1. As seen in the proof of Lemma 3.4. the density $p_{\theta}$ exists in the Schwartz space. Note that for a function $f$ in the Schwartz space, by Taylor's theorem,

$$
\begin{aligned}
\left|f(x+a)-f(x)-f^{\prime}(x) a-f^{\prime \prime}(x) \frac{a^{2}}{2}\right| & \leq \frac{a^{3}}{2} \sup _{|b| \leq|a|}\left|f^{\prime \prime \prime}(x+b)\right| \\
& \leq \frac{a^{3}}{2} \sup _{|b| \leq|a|} \frac{1}{\left(1+(x+b)^{2}\right)^{\alpha}} \sup _{y \in \mathbb{R}}\left(1+y^{2}\right)^{\alpha}\left|f^{\prime \prime \prime}(y)\right|
\end{aligned}
$$

and so,

$$
\sup _{x \in \mathbb{R}}\left(1+x^{2}\right)^{\alpha}\left|f(x+a)-f(x)-f^{\prime}(x) a-f^{\prime \prime}(x) \frac{a^{2}}{2}\right|=O\left(a^{3}\right) .
$$

This gives

$$
\sup _{x \in \mathbb{R}}\left(1+x^{2}\right)^{\alpha}\left|q_{\theta}(x)-\bar{q}_{\theta}(x)\right|=O\left(\theta^{1+H}\right)=o\left(\theta^{2 H}\right),
$$

where $\bar{q}_{\theta}$ is given by $[8$. By the Fourier identity,

$$
\left(1+x^{2}\right)^{\alpha}\left(p_{\theta}(x)-\bar{q}_{\theta}(x)\right)=\frac{1}{2 \pi} \iint e^{i u y}\left(1+y^{2}\right)^{\alpha}\left(p_{\theta}(y)-\bar{q}_{\theta}(y)\right) \mathrm{d} y e^{-i u x} \mathrm{~d} u
$$

Combining the lemmas in the previous section, taking $\delta \in(0, \min \{\epsilon,(H-\epsilon) / 3\})$, we have

$$
\int_{|u| \leq \theta^{-\delta}}\left|\int e^{i u y}\left(1+y^{2}\right)^{\alpha}\left(p_{\theta}(y)-\bar{q}_{\theta}(y)\right) \mathrm{d} y\right| \mathrm{d} u=o\left(\theta^{2 H}\right) .
$$

On the other hand,

$$
\begin{aligned}
\int_{|u| \geq \theta^{-\delta}}\left|\int e^{i u y}\left(1+y^{2}\right)^{\alpha} p_{\theta}(y) \mathrm{d} y\right| \mathrm{d} u & \leq \theta^{j \delta} \int_{|u| \geq \theta^{-\delta}}|u|^{j}\left|E\left[\left(1+X_{\theta}^{2}\right)^{\alpha} e^{i u X_{\theta}}\right]\right| \mathrm{d} u \\
& =O\left(\theta^{j \delta}\right)
\end{aligned}
$$

for any $j \in \mathbb{N}$ by Lemma 3.4 . The remainder

$$
\int_{|u| \geq \theta^{-\delta}}\left|\int e^{i u y}\left(1+y^{2}\right)^{\alpha} \bar{q}_{\theta}(y) \mathrm{d} y\right| \mathrm{d} u
$$

is handled in the same manner.

\subsection{Put option price expansion}

Here we consider put option prices. Denote by $p_{\theta}$ the density of $X_{\theta}$ as before and consider a normalized put option price

$$
\frac{p\left(F e^{\sigma_{0}(\theta) z}, \theta\right)}{F \sigma_{0}(\theta)}=e^{-r \theta} \int_{-\infty}^{z} \int_{-\infty}^{\zeta} p_{\theta}(x) \mathrm{d} x e^{\sigma_{0}(\theta) \zeta} \mathrm{d} \zeta
$$


Lemma 3.5 Let $q_{\theta}(x), \theta>0$ be a family of functions on $\mathbb{R}$ (not necessarily the one given by (8)). If

$$
\sup _{x \in \mathbb{R}}\left(1+x^{2}\right)^{\alpha}\left|p_{\theta}(x)-q_{\theta}(x)\right|=o\left(\theta^{\beta}\right)
$$

for some $\alpha>5 / 4$ and $\beta>0$, then for any $z_{0} \in \mathbb{R}$,

$$
\frac{p\left(F e^{\sigma_{0}(\theta) z}, \theta\right)}{F \sigma_{0}(\theta)}=e^{-r \theta} \int_{-\infty}^{z} \int_{-\infty}^{\zeta} q_{\theta}(x) \mathrm{d} x e^{\sigma_{0}(\theta) \zeta} \mathrm{d} \zeta+o\left(\theta^{\beta}\right)
$$

uniformly in $z \leq z_{0}$.

Proof: By the Cauchy-Schwarz inequality,

$$
\begin{aligned}
& e^{-r \theta} \int_{-\infty}^{z} \int_{-\infty}^{\zeta}\left|p_{\theta}(x)-q_{\theta}(x)\right| \mathrm{d} z e^{\sigma_{0}(\theta) \zeta} \mathrm{d} \zeta \\
& \leq e^{-r \theta} \int_{-\infty}^{z} \sqrt{\int_{-\infty}^{\zeta} \frac{\mathrm{d} x}{\left(1+x^{2}\right)^{2 \alpha-1}}} \sqrt{\int_{-\infty}^{\zeta}\left(1+x^{2}\right)^{2 \alpha-1}\left|p_{\theta}(x)-q_{\theta}(x)\right|^{2} \mathrm{~d} z e^{\sigma_{0}(\theta) \zeta} \mathrm{d} \zeta} \\
& \leq \sqrt{\pi} e^{-r \theta+\sigma_{0}(\theta) z} \sup _{x \in \mathbb{R}}\left(1+x^{2}\right)^{\alpha}\left|p_{\theta}(x)-q_{\theta}(x)\right| \int_{-\infty}^{z} \sqrt{\int_{-\infty}^{\zeta} \frac{\mathrm{d} x}{\left(1+x^{2}\right)^{2 \alpha-1}}} \mathrm{~d} \zeta,
\end{aligned}
$$

which is $o\left(\theta^{\beta}\right)$ if $\alpha>5 / 4$.

Proof of Theorem 2.2. This is a direct consequence of the previous lemma. For example,

$$
\frac{\mathrm{d}}{\mathrm{d} z}\left\{e^{-\sigma_{0}(\theta) z} \frac{\mathrm{d}}{\mathrm{d} z}\left\{\frac{1}{\sigma_{0}(\theta)}\left(\Phi\left(z+\frac{\sigma_{0}(\theta)}{2}\right) e^{\sigma_{0}(\theta) z}-\Phi\left(z-\frac{\sigma_{0}(\theta)}{2}\right)\right)\right\}\right\}=\phi\left(z+\frac{\sigma_{0}(\theta)}{2}\right)
$$

The derivative of $H_{k}(z) \phi(z)$ is $-H_{k+1}(z) \phi(z)$. Recall also $\sigma_{0}(\theta)=O(\sqrt{\theta})$. ////

\subsection{Implied volatility expansion}

Here we prove an expansion formula for the Black-Scholes implied volatility.

Proof of Theorem 2.3. Step 1). Fix $z \in \mathbb{R}$. Note that

$$
P_{\theta}(\sigma):=\frac{p_{\mathrm{BS}}\left(F e^{\sqrt{\theta} z}, \theta, \sigma\right)}{F e^{-r \theta} \sqrt{\theta}}=\frac{1}{\sqrt{\theta}}\left(\Phi\left(\frac{z}{\sigma}+\frac{\sigma \sqrt{\theta}}{2}\right) e^{\sqrt{\theta} z}-\Phi\left(\frac{z}{\sigma}-\frac{\sigma \sqrt{\theta}}{2}\right)\right)
$$

and that

$$
P_{\theta}:[0, \infty] \rightarrow\left[\frac{\left(e^{\sqrt{\theta} z}-1\right)_{+}}{\sqrt{\theta}}, \frac{e^{\sqrt{\theta} z}}{\sqrt{\theta}}\right]
$$


is a strictly increasing function. From (9) and Proposition 2.2, we have

$$
\begin{aligned}
\frac{p\left(F e^{\sqrt{\theta} z}, \theta\right)}{F e^{-r \theta} \sqrt{\theta}}= & P_{\theta}\left(\kappa_{2}\right)+\kappa_{2} \kappa_{3} \phi\left(\frac{z}{\kappa_{2}}+\frac{\kappa_{2} \sqrt{\theta}}{2}\right) H_{1}\left(\frac{z}{\kappa_{2}}+\frac{\kappa_{2} \sqrt{\theta}}{2}\right) e^{\sqrt{\theta} z} \theta^{H} \\
& +\kappa_{2} \phi\left(\frac{z}{\kappa_{2}}\right)\left(\kappa_{4} H_{2}\left(\frac{z}{\kappa_{2}}\right)+\frac{\kappa_{3}^{2}}{2} H_{4}\left(\frac{z}{\kappa_{2}}\right)\right) \theta^{2 H}+o\left(\theta^{2 H}\right) \\
= & P \theta\left(\kappa_{2}\right)+O\left(\theta^{H}\right) .
\end{aligned}
$$

Therefore

$$
\sigma_{\mathrm{BS}}(\sqrt{\theta} z, \theta)=P_{\theta}^{-1}\left(P_{\theta}\left(\kappa_{2}\right)+O\left(\theta^{H}\right)\right) .
$$

By (1), $\kappa_{2}$ is bounded in $\theta$, say, by $L>0$. The function $P_{\theta}$ converges as $\theta \rightarrow 0$ to

$$
P_{0}(\sigma):=z \Phi\left(\frac{z}{\sigma}\right)+\sigma \phi\left(\frac{z}{\sigma}\right)
$$

pointwise, and by Dini's theorem, this convergence is uniform on $[0, L]$. Since the limit function $P_{0}$ is strictly increasing, the inverse functions $P_{\theta}^{-1}$ converges to $P_{0}^{-1}$. Again by Dini's theorem, this convergence is uniform and in particular, $P_{\theta}^{-1}$ are equicontinuous. Thus we conclude $\sigma_{\mathrm{BS}}(\sqrt{\theta} z, \theta)-\kappa_{2} \rightarrow 0$ as $\theta \rightarrow 0$. Then, write $\sigma_{\mathrm{BS}}(\sqrt{\theta} z, \theta)=\kappa_{2}+\beta(\theta)$ and substitute this to the equation $P_{\theta}\left(\sigma_{\mathrm{BS}}(\sqrt{\theta} z, \theta)\right)=P_{\theta}\left(\kappa_{2}\right)+O\left(\theta^{H}\right)$. The Taylor expansion gives $\beta(\theta)=O\left(\theta^{H}\right)$.

Step 2). From (9) we have

$$
P_{\theta}(\sigma)=\sigma F_{1}\left(\frac{z}{\sigma}\right)+\frac{\sigma^{2} \sqrt{\theta}}{2} F_{2}\left(\frac{z}{\sigma}\right)+\frac{\sigma^{3} \theta}{6} F_{3}\left(\frac{z}{\sigma}\right)+o(\theta),
$$

where

$$
F_{1}(x)=x \Phi(x)+\phi(x), \quad F_{2}(x)=x^{2} \Phi(x)+x \phi(x), \quad F_{3}(x)=x^{3} \Phi(x)+\left(x^{2}-\frac{1}{4}\right) \phi(x) .
$$

Using that

$$
\partial_{\sigma}\left\{\sigma F_{1}\left(\frac{z}{\sigma}\right)\right\}=\phi\left(\frac{z}{\sigma}\right)
$$

we have

$$
\begin{aligned}
& \kappa_{2} F_{1}\left(\frac{z}{\kappa_{2}}\right)+\frac{\kappa_{2}^{2} \sqrt{\theta}}{2} F_{2}\left(\frac{z}{\kappa_{2}}\right)+\kappa_{2} \phi\left(\frac{z}{\kappa_{2}}\right) \kappa_{3} H_{1}\left(\frac{z}{\kappa_{2}}\right) e^{\sqrt{\theta} z} \theta^{H} \\
& =\sigma_{\mathrm{BS}}(\sqrt{\theta} z, \theta) F_{1}\left(\frac{z}{\sigma_{\mathrm{BS}}(\sqrt{\theta} z, \theta)}\right)+\frac{\sigma_{\mathrm{BS}}(\sqrt{\theta} z, \theta)^{2} \sqrt{\theta}}{2} F_{2}\left(\frac{z}{\sigma_{\mathrm{BS}}(\sqrt{\theta} z, \theta)}\right)+O\left(\theta^{2 H}\right) \\
& =\kappa_{2} F_{1}\left(\frac{z}{\kappa_{2}}\right)+\frac{\kappa_{2}^{2} \sqrt{\theta}}{2} F_{2}\left(\frac{z}{\kappa_{2}}\right)+\phi\left(\frac{z}{\kappa_{2}}\right)\left(\sigma_{\mathrm{BS}}(\sqrt{\theta} z, \theta)-\kappa_{2}\right)+O\left(\theta^{2 H}\right)
\end{aligned}
$$

from which we conclude $\sigma_{\mathrm{BS}}(\sqrt{\theta} z, \theta)=\kappa_{2}+\kappa_{3} z e^{\sqrt{\theta} z} \theta^{H}+O\left(\theta^{2 H}\right)$. 
Step 3). Using that

$$
\partial_{\sigma}^{2}\left\{\sigma F_{1}\left(\frac{z}{\sigma}\right)\right\}=\frac{z^{2}}{\sigma^{3}} \phi\left(\frac{z}{\sigma}\right), \partial_{\sigma}\left\{\sigma^{2} F_{2}\left(\frac{z}{\sigma}\right)\right\}=z \phi\left(\frac{z}{\sigma}\right),
$$

we obtain

$$
\begin{aligned}
\kappa_{2} \phi & \left(\frac{z}{\kappa_{2}}+\frac{\kappa_{2} \sqrt{\theta}}{2}\right)\left(\kappa_{3} H_{1}\left(\frac{z}{\kappa_{2}}+\frac{\kappa_{2} \sqrt{\theta}}{2}\right) e^{\sqrt{\theta} z} \theta^{H}+\left(\kappa_{4} H_{2}\left(\frac{z}{\kappa_{2}}\right)+\frac{\kappa_{3}^{2}}{2} H_{4}\left(\frac{z}{\kappa_{2}}\right)\right) \theta^{2 H}\right) \\
= & \frac{p\left(F e^{\sqrt{\theta} z}, \theta\right)}{F e^{-r \theta} \sqrt{\theta}}-P_{\theta}\left(\kappa_{2}\right)+o\left(\theta^{2 H}\right) \\
= & P_{\theta}\left(\sigma_{\mathrm{BS}}(\sqrt{\theta} z, z)\right)-P_{\theta}\left(\kappa_{2}\right)+o\left(\theta^{2 H}\right) \\
= & \left.\partial_{\sigma}\left\{\sigma F_{1}\left(\frac{z}{\sigma}\right)\right\}\right|_{\sigma=\kappa_{2}}\left(\sigma_{\mathrm{BS}}(\sqrt{\theta} z, \theta)-\kappa_{2}\right)+\left.\frac{1}{2} \partial_{\sigma}^{2}\left\{\sigma F_{1}\left(\frac{z}{\sigma}\right)\right\}\right|_{\sigma=\kappa_{2}}\left(\sigma_{\mathrm{BS}}(\sqrt{\theta} z, \theta)-\kappa_{2}\right)^{2} \\
& +\left.\frac{\sqrt{\theta}}{2} \partial_{\sigma}\left\{\sigma^{2} F_{2}\left(\frac{z}{\sigma}\right)\right\}\right|_{\sigma=\kappa_{2}}\left(\sigma_{\mathrm{BS}}(\sqrt{\theta} z, \theta)-\kappa_{2}\right)+o\left(\theta^{2 H}\right) \\
= & \phi\left(\frac{z}{\kappa_{2}}\right)\left(\sigma_{\mathrm{BS}}(\sqrt{\theta} z, \theta)-\kappa_{2}\right)+\frac{\sqrt{\theta}}{2} z \phi\left(\frac{z}{\kappa_{2}}\right)\left(\sigma_{\mathrm{BS}}(\sqrt{\theta} z, \theta)-\kappa_{2}\right) \\
& +\frac{z^{2}}{2 \kappa_{2}^{3}} \phi\left(\frac{z}{\kappa_{2}}\right)\left(\sigma_{\mathrm{BS}}(\sqrt{\theta} z, \theta)-\kappa_{2}\right)^{2}+o\left(\theta^{2 H}\right)
\end{aligned}
$$

from Theorem 2.2 and Step 2. The left hand side is further expanded as

$$
\begin{aligned}
\kappa_{2} \phi\left(\frac{z}{\kappa_{2}}\right)\left\{\kappa_{3} H_{1}\left(\frac{z}{\kappa_{2}}\right) e^{\sqrt{\theta} z} \theta^{H}-\kappa_{3} H_{2}\left(\frac{z}{\kappa_{2}}\right) \frac{\kappa_{2}}{2} \theta^{H+1 / 2}\right. & \\
& \left.+\left(\kappa_{4} H_{2}\left(\frac{z}{\kappa_{2}}\right)+\frac{\kappa_{3}^{2}}{2} H_{4}\left(\frac{z}{\kappa_{2}}\right)\right) \theta^{2 H}\right\}+o\left(\theta^{2 H}\right) .
\end{aligned}
$$

Denote $\gamma(\theta)=\sigma_{\mathrm{BS}}(\sqrt{\theta} z, \theta)-\kappa_{2}-\kappa_{3} z e^{\sqrt{\theta} z} \theta^{H}$ and substitute this to obtain

$$
\begin{aligned}
\gamma(\theta)= & -\kappa_{3} H_{2}\left(\frac{z}{\kappa_{2}}\right) \frac{\kappa_{2}^{2}}{2} \theta^{H+1 / 2}+\kappa_{2}\left(\kappa_{4} H_{2}\left(\frac{z}{\kappa_{2}}\right)+\frac{\kappa_{3}^{2}}{2} H_{4}\left(\frac{z}{\kappa_{2}}\right)\right) \theta^{2 H} \\
& -\frac{\kappa_{3}}{2} z^{2} \theta^{H+1 / 2}-\frac{\kappa_{3}^{2}}{2 \kappa_{2}^{3}} z^{4} \theta^{2 H}+o\left(\theta^{2 H}\right) \\
= & \left(\frac{\kappa_{2}^{2}}{2}-z^{2}\right) \kappa_{3} \theta^{H+1 / 2}+\kappa_{2}\left(\left(\kappa_{4}-3 \kappa_{3}^{2}\right) \frac{z^{2}}{\kappa_{2}^{2}}+\frac{3}{2} \kappa_{3}^{2}-\kappa_{4}\right) \theta^{2 H}+o\left(\theta^{2 H}\right),
\end{aligned}
$$

from which we conclude the result.

\subsection{Asymptotics for at-the-money skew and curvature}

Here we prove Theorem 2.4 
Proof of Theorem 2.4 It is known (see e.g., Fukasawa [9]) that

$$
\begin{aligned}
& \partial_{k} \sigma_{\mathrm{BS}}(k, \theta)=\frac{Q\left(k \geq \sigma_{0}(\theta) X_{\theta}\right)-\Phi\left(f_{2}(k, \theta)\right)}{\sqrt{\theta} \phi\left(f_{2}(k, \theta)\right)}, \\
& \partial_{k}^{2} \sigma_{\mathrm{BS}}(k, \theta)=\frac{p_{\theta}\left(k / \sigma_{0}(\theta)\right)}{\sigma_{0}(\theta) \sqrt{\theta} \phi\left(f_{2}(k, \theta)\right)}-\sigma_{\mathrm{BS}}(k, \theta) \partial_{k} f_{1}(k, \theta) \partial_{k} f_{2}(k, \theta),
\end{aligned}
$$

where

$$
f_{1}(k, \theta)=\frac{k}{\sqrt{\theta} \sigma_{\mathrm{BS}}(k, \theta)}-\frac{\sqrt{\theta} \sigma_{\mathrm{BS}}(k, \theta)}{2}, f_{2}(k, \theta)=\frac{k}{\sqrt{\theta} \sigma_{\mathrm{BS}}(k, \theta)}+\frac{\sqrt{\theta} \sigma_{\mathrm{BS}}(k, \theta)}{2} \text {. }
$$

Since the condition of Theorem 2.2 is met, we have

$$
Q\left(0 \geq X_{\theta}\right)=\Phi\left(\frac{\sigma_{0}(\theta)}{2}\right)+\kappa_{3}(\theta) \phi\left(\frac{\sigma_{0}(\theta)}{2}\right) \theta^{H}+o\left(\theta^{2 H}\right) .
$$

On the other hand, by Theorem 2.3 .

$$
f_{2}(0, \theta)=\frac{\sqrt{\theta}}{2} \kappa_{2}(\theta)+O\left(\theta^{2 H+1 / 2}\right)
$$

and so,

$$
\begin{aligned}
& \Phi\left(f_{2}(0, \theta)\right)=\Phi\left(\frac{\sigma_{0}(\theta)}{2}\right)+O\left(\theta^{2 H+1 / 2}\right), \\
& \phi\left(f_{2}(0, \theta)\right)=\phi(0)-\phi(0) \frac{\theta}{8} \kappa_{2}(\theta)^{2}+O\left(\theta^{2 H+1}\right) .
\end{aligned}
$$

Then, it follows from (10) that

$$
\partial_{k} \sigma_{\mathrm{BS}}(0, \theta)=\kappa_{3}(\theta) \theta^{H-1 / 2}+o\left(\theta^{2 H-1 / 2}\right) .
$$

Further, under the condition, we have

$$
p_{\theta}(0)=\phi\left(\frac{\sigma_{0}(\theta)}{2}\right)\left\{1-\frac{\kappa_{3}(\theta)}{2} \sigma_{0}(\theta) \theta^{H}+\left(3 \kappa_{4}(\theta)-15 \frac{\kappa_{3}(\theta)^{2}}{2}\right) \theta^{2 H}\right\}+o\left(\theta^{2 H}\right) .
$$

On the other hand, by Theorem 2.3 and (11),

$$
\begin{aligned}
\sigma_{\mathrm{BS}}(0, \theta) & \partial_{k} f_{1}(0, \theta) \partial_{k} f_{2}(0, \theta) \\
& =\frac{1}{\sigma_{\mathrm{BS}}(0, \theta) \theta}+O\left(\theta^{2 H}\right) \\
& =\frac{1}{\kappa_{2}(\theta) \theta}\left(1-\frac{1}{2} \kappa_{2}(\theta) \kappa_{3}(\theta) \theta^{H+1 / 2}-\left(\frac{3}{2} \kappa_{3}(\theta)^{2}-\kappa_{4}(\theta)\right) \theta^{2 H}\right)+o\left(\theta^{2 H-1}\right) .
\end{aligned}
$$

Then, it follows from (10) that

$$
\partial_{k}^{2} \sigma_{\mathrm{BS}}(0, \theta)=\frac{2 \kappa_{4}(\theta)-6 \kappa_{3}(\theta)^{2}}{\kappa_{2}(\theta)} \theta^{2 H-1}+o\left(\theta^{2 H-1}\right),
$$

which completes the proof. 


\section{Regular stochastic volatility models}

Here we briefly discuss that regular stochastic volatility models satisfy all the assumptions in Section 2.1 with $H=1 / 2$. Consider the volatility process $v_{t}=v\left(X_{t}\right)$, where $X$ is a Markov process satisfying a stochastic differential equation

$$
\mathrm{d} X_{t}=b\left(X_{t}\right) \mathrm{d} t+c\left(X_{t}\right) \mathrm{d} W_{t}
$$

and $v$ is a smooth positive function defined on the state space of $X$. Let $\rho \in(-1,1)$ be a constant and $\left\{\mathcal{G}_{t}\right\}$ be the augmented filtration generated by $W$. We assume (1), which is satisfied in the usual cases including the log-normal SABR and Heston models. Denote by $L$ the generator of $X$. Put $f=\sqrt{v}, g=f^{\prime} c$ and $h=v^{\prime} c$. Then, by Itô's formula, we have

$$
\begin{gathered}
M_{\theta}=f\left(X_{0}\right) B_{\theta}+\int_{0}^{\theta} \int_{0}^{t} g\left(X_{s}\right) \mathrm{d} W_{s} \mathrm{~d} B_{t}+\int_{0}^{\theta} \int_{0}^{t} L f\left(X_{s}\right) \mathrm{d} s \mathrm{~d} B_{t}, \\
\langle M\rangle_{\theta}=v\left(X_{0}\right) \theta+\int_{0}^{\theta} \int_{0}^{t} h\left(X_{s}\right) \mathrm{d} W_{s} \mathrm{~d} t+\int_{0}^{\theta} \int_{0}^{t} L v\left(X_{s}\right) \mathrm{d} s \mathrm{~d} t .
\end{gathered}
$$

Let $\bar{B}_{t}^{\theta}=\theta^{-1 / 2} B_{\theta t}, \bar{W}_{t}^{\theta}=\theta^{-1 / 2} W_{\theta t}$ and $X_{t}^{\theta}=X_{\theta t}$. Then

$$
\begin{aligned}
& \frac{M_{\theta}}{\sqrt{\theta}}=f\left(X_{0}\right) \bar{B}_{1}^{\theta}+\sqrt{\theta} \int_{0}^{1} \int_{0}^{u} g\left(X_{v}^{\theta}\right) \mathrm{d} \bar{W}_{v}^{\theta} \mathrm{d} \bar{B}_{u}^{\theta}+\theta \int_{0}^{1} \int_{0}^{u} L f\left(X_{v}^{\theta}\right) \mathrm{d} v \mathrm{~d} \bar{B}_{u}^{\theta}, \\
& \frac{\langle M\rangle_{\theta}}{\theta}=v\left(X_{0}\right)+\sqrt{\theta} \int_{0}^{1} \int_{0}^{u} h\left(X_{v}^{\theta}\right) \mathrm{d} \bar{W}_{v}^{\theta} \mathrm{d} u+\theta \int_{0}^{1} \int_{0}^{u} L v\left(X_{v}^{\theta}\right) \mathrm{d} v \mathrm{~d} u .
\end{aligned}
$$

It would follow that

$$
\frac{\sigma_{0}(\theta)^{2}}{\theta}=\frac{E\left[\langle M\rangle_{\theta}\right]}{\theta}=v\left(X_{0}\right)+\frac{1}{2} L v\left(X_{0}\right) \theta+O\left(\theta^{3 / 2}\right),
$$

and so

$$
\frac{\sigma_{0}(\theta)}{\sqrt{\theta}}=f\left(X_{0}\right)+\frac{1}{4} \frac{L v\left(X_{0}\right)}{f\left(X_{0}\right)} \theta+O\left(\theta^{3 / 2}\right)
$$

under a mild regularity condition. Then, we have (3) with $H=1 / 2, M_{\theta}^{(0)}=\bar{B}_{1}^{\theta}$ and

$$
\begin{aligned}
& M_{\theta}^{(1)}=\frac{g\left(X_{0}\right)}{f\left(X_{0}\right)} \int_{0}^{1} \bar{W}_{u}^{\theta} \mathrm{d} \bar{B}_{u}^{\theta}, \\
& M_{\theta}^{(2)}=-\frac{L v\left(X_{0}\right)}{4 v\left(X_{0}\right)} \bar{B}_{1}^{\theta}+\frac{g^{\prime}\left(X_{0}\right) c\left(X_{0}\right)}{f\left(X_{0}\right)} \int_{0}^{1} \int_{0}^{u} \bar{W}_{v}^{\theta} \mathrm{d} \bar{W}_{v}^{\theta} \mathrm{d} \bar{B}_{u}^{\theta}+\frac{L f\left(X_{0}\right)}{f\left(X_{0}\right)} \int_{0}^{1} u \mathrm{~d} \bar{B}_{u}^{\theta}, \\
& M_{\theta}^{(3)}=2 \frac{g\left(X_{0}\right)}{f\left(X_{0}\right)} \int_{0}^{1} \bar{W}_{u}^{\theta} \mathrm{d} u
\end{aligned}
$$

again under a mild regularity condition. By Nualart et al. [18] or Appendix A below,

$$
E\left[M_{\theta}^{(1)} \mid M_{\theta}^{(0)}=x\right]=\frac{g\left(X_{0}\right)}{f\left(X_{0}\right)} \frac{\rho}{2} H_{2}(x), \quad E\left[M_{\theta}^{(3)} \mid M_{\theta}^{(0)}=x\right]=\frac{g\left(X_{0}\right)}{f\left(X_{0}\right)} \rho H_{1}(x)
$$


and so,

$$
\begin{aligned}
a_{\theta}^{(1)} & \left(x+\frac{\sigma_{0}(\theta)}{2}\right)-\frac{\sigma_{0}(\theta)}{2} a_{\theta}^{(3)}\left(x+\frac{\sigma_{0}(\theta)}{2}\right) \\
& =-\kappa_{3}\left(H_{3}\left(x+\frac{\sigma_{0}(\theta)}{2}\right)-\sigma_{0}(\theta) H_{2}\left(x+\frac{\sigma_{0}(\theta)}{2}\right)\right) \phi\left(x+\frac{\sigma_{0}(\theta)}{2}\right)
\end{aligned}
$$

with

$$
\kappa_{3}=\frac{\rho}{2} \frac{g\left(X_{0}\right)}{f\left(X_{0}\right)} .
$$

Further,

$$
\begin{aligned}
E\left[M_{\theta}^{(2)} \mid M_{\theta}^{(0)}=x\right] & =-\frac{L v}{4 v}\left(X_{0}\right) x+\frac{g^{\prime} c}{f}\left(X_{0}\right) \frac{\rho^{2}}{6} H_{3}(x)+\frac{L f}{2 f}\left(X_{0}\right) x \\
& =-\frac{g^{2}}{4 f^{2}}\left(X_{0}\right) x+\frac{g^{\prime} c}{f}\left(X_{0}\right) \frac{\rho^{2}}{6} H_{3}(x)
\end{aligned}
$$

and

$$
E\left[\left(M_{\theta}^{(1)}\right)^{2} \mid M_{\theta}^{(0)}=x\right]=\frac{g^{2}}{f^{2}}\left(X_{0}\right)\left(\rho^{2}\left(\frac{1}{2}+H_{2}(x)+\frac{1}{4} H_{4}(x)\right)+\left(1-\rho^{2}\right)\left(\frac{1}{2}+\frac{1}{3} H_{2}(x)\right)\right) .
$$

Therefore,

$$
a_{\theta}^{(2)}(x)-\frac{1}{2} c_{\theta}(x)=-\kappa_{4} H_{4}(x) \phi(x)-\frac{\kappa_{3}^{2}}{2} H_{6}(x) \phi(x)
$$

with

$$
\kappa_{4}=\frac{g^{\prime} c}{f}\left(X_{0}\right) \frac{\rho^{2}}{6}+\frac{g^{2}}{f^{2}}\left(X_{0}\right) \frac{1+2 \rho^{2}}{6} .
$$

Thus we have observed that (6) has the form of (7) and so, all the theorems in Section 2.2 are applied. In particular, Theorem 2.3 proves the Medvedev-Scaillet formula (Proposition 1 of [16] that was obtained by a formal computation when $f$ is the identity function).

\section{The rough Bergomi model}

Here we show that the rough Bergomi model proposed by [3] fits into the framework and compute the expansion terms. Let $\rho_{t}=\rho \in(-1,1)$ be a constant and

$$
v_{t}=v_{0}(t) \exp \left\{\eta \sqrt{2 H} \int_{0}^{t}(t-s)^{H-1 / 2} \mathrm{~d} W_{s}-\frac{\eta^{2}}{2} t^{2 H}\right\} .
$$

The deterministic function $v_{0}(t)=E\left[v_{t}\right]$ is assumed to be continuous. 
Theorem 5.1 We have (5) for $q_{\theta}$ given by (7) with

$$
\begin{aligned}
& \kappa_{3}(\theta)=\rho \eta \sqrt{\frac{H}{2}} \frac{1}{\theta^{H} \sigma_{0}(\theta)^{3}} \int_{0}^{\theta} \int_{0}^{t}(t-s)^{H-1 / 2} \sqrt{v_{0}(s)} \mathrm{d} s v_{0}(t) \mathrm{d} t, \\
& \kappa_{4}(\theta)=\frac{\left(1+2 \rho^{2}\right) \eta^{2} H}{(2 H+1)^{2}(2 H+2)}+\frac{\rho^{2} \eta^{2} H \beta(H+3 / 2, H+3 / 2)}{2(H+1 / 2)^{2}},
\end{aligned}
$$

where $\beta$ is the beta function.

Proof: Since $v_{t}$ is log-normally distributed, (1) holds by Jensen's inequality. The conditions (2) and (3) follow from Lemma 5.1 below. The functions $a_{\theta}^{(i)}$ and $c_{\theta}$ are computed in Lemmas 5.2, 5.3 5.4 and 5.5 below. The function $b_{\theta}$ is obtained as the the derivative of $a_{\theta}^{(1)}$. They are apparently rapidly decreasing smooth functions. Then, by Theorem 2.1. it suffices to show that $q_{\theta}$ defined by (8) has the form (7) up to $o\left(\theta^{2 H}\right)$ with $\kappa_{3}(\theta)$ and $\kappa_{4}(\theta)$ specified above.

Theorems 2.2, 2.3 and 2.4 are therefore valid here as well. When $H<1 / 2$ and the forward variance curve is flat (i.e., $v_{0}$ is constant), Theorem 2.3 gives a similar formula to the Bergomi-Guyon expansion formally derived in [3] $]^{2}$ In fact, the expansion of $O(\eta)$ given in [3] coincides with our expansion of $O\left(\theta^{H}\right)$ when $v_{0}$ is constant. When $v_{0}$ is not constant, or when looking at the secondorder terms, the formulas are not the same; this is not surprising because the asymptotics are $\eta \rightarrow 0$ in $|3|$ while $\theta \rightarrow 0$ here. Further, when $v_{0}$ is constant, the same formula of $O\left(\theta^{H}\right)$ can be obtained by expanding the rate function of the large deviation result of [6] as in [4]. To be more precise, note that by Theorem 5.1.

$$
\kappa_{3}(\theta)=\rho \frac{\eta \sqrt{2 H}}{2(H+1 / 2)(H+3 / 2)}
$$

when $v_{0}$ is constant and Theorem 2.3 implies

$$
\frac{\sigma_{\mathrm{BS}}(\sqrt{\theta} z, \theta)-\sigma_{\mathrm{BS}}(\sqrt{\theta} \zeta, \theta)}{\sqrt{\theta} z-\sqrt{\theta} \zeta}=\kappa_{3}(\theta) \theta^{H-1 / 2}+O\left(\theta^{2 H-1 / 2}\right)
$$

for $z \neq \zeta$. A weaker assertion, where $O\left(\theta^{2 H-1 / 2}\right)$ is replaced with $o\left(\theta^{H-1 / 2}\right)$, was already shown in [10] by a different method. What is shown in [4] via an expansion of the rate function is that this formula up to $o\left(\theta^{H-1 / 2}\right)$ remains valid even if $\sqrt{\theta} z$ and $\sqrt{\theta} \zeta$ are replaced with $\theta^{\beta} z$ and $\theta^{\beta} \zeta$ respectively for $\beta \in(1 / 2-H, 1 / 2)$.

How small $\theta$ has to be for reasonable accuracy of our asymptotic formulas should be examined via numerical experiments. Our extensive experiments suggest $\eta \theta^{H}<1$ as a rough criterion ${ }^{3}$. Here we present only a few examples of the volatility surfaces. In Figures 1 and 2 , the points are by the Monte Carlo

\footnotetext{
${ }^{2}$ Note however that there is a typo in the second order term in [3].

${ }^{3}$ Note that $\eta \theta^{H}$ is the standard deviation of log-spot-variance.
} 

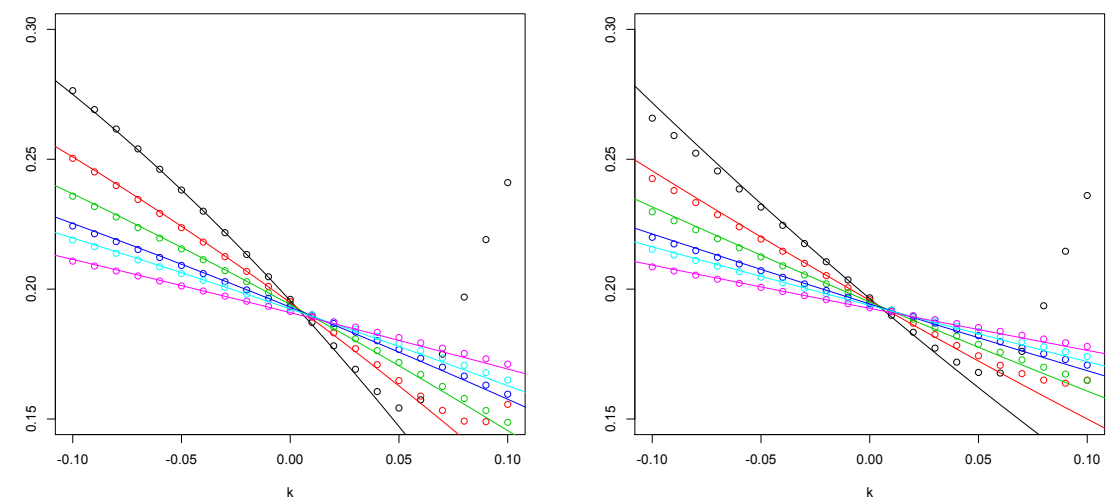

Figure 1: The Black-Scholes implied volatility under the rough Bergomi model with $v_{0} \equiv .04$ and $(H, \rho, \eta)=(.07,-.9, .9)$ (left) or $(H, \rho, \eta)=(.07,-.7, .9)$ (right).
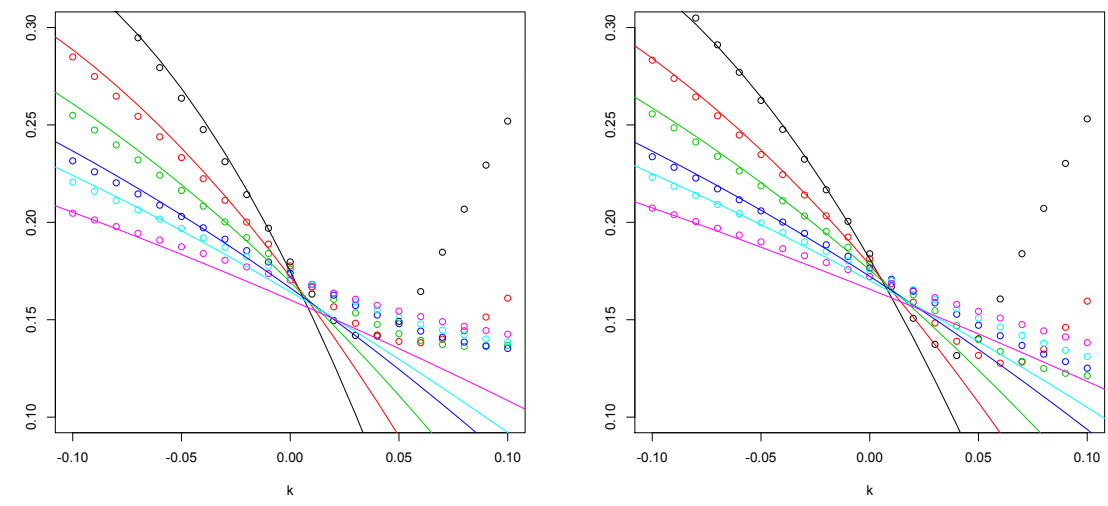

Figure 2: The Black-Scholes implied volatility under the rough Bergomi model with $v_{0} \equiv .04$ and $(H, \rho, \eta)=(.05,-.9,2.3)$ (left) or $(H, \rho, \eta)=(.07,-.9,1.9)$ (right). 
and the curves are by the asymptotic formula given in Theorems 2.3 and 5.1 The different colors are for different time-to-maturities; black for $\theta=.02$, red for $\theta=.05$, green for $\theta=.1$, blue for $\theta=.2$, cyan for $\theta=.3$ and magenta for $\theta=.6$. Note that the sets of parameters in Figure 2 are those calibrated from option data by Bayer et al [3].

In order to prove Lemmas below, we need some preparation. Let $H_{k}, k=$ $0,1, \ldots$ be the Hermite polynomials as before:

$$
H_{k}(x)=(-1)^{k} e^{x^{2} / 2} \frac{\mathrm{d}^{k}}{\mathrm{~d} x^{k}} e^{-x^{2} / 2}
$$

and $H_{k}(x, a)=a^{k / 2} H_{k}(x / \sqrt{a})$ for $a>0$. As is well-known, we have

$$
\exp \left\{u x-\frac{a u^{2}}{2}\right\}=\sum_{k=0}^{\infty} H_{k}(x, a) \frac{u^{k}}{k !}
$$

and for any continuous local martingale $M$ and $n \in \mathbb{N}$,

$$
\mathrm{d} L_{t}^{(n)}=n L_{t}^{(n-1)} \mathrm{d} M_{t}
$$

where $L^{(k)}=H_{k}(M,\langle M\rangle)$ for $k \in \mathbb{N}$. See, e.g., Revuz and Yor [21].

Define $\hat{W}, \hat{W}^{\prime}, \hat{B}$ by

$$
\hat{W}_{t}=\frac{1}{\sigma_{0}(\theta)} \int_{0}^{\tau^{-1}(t)} \sqrt{v_{0}(s)} \mathrm{d} W_{s}, \quad \hat{W}_{t}^{\prime}=\frac{1}{\sigma_{0}(\theta)} \int_{0}^{\tau^{-1}(t)} \sqrt{v_{0}(s)} \mathrm{d} W_{s}^{\prime}
$$

and $\hat{B}=\rho \hat{W}+\sqrt{1-\rho^{2}} \hat{W}^{\prime}$, where

$$
\tau(s)=\frac{1}{\sigma_{0}(\theta)^{2}} \int_{0}^{s} v_{0}(t) \mathrm{d} t .
$$

Then, $\left(\hat{W}, \hat{W}^{\prime}\right)$ is a 2-dimensional Brownian motion under $E$ and for any squareintegrable function $f$,

$$
\int_{0}^{a} f(s) \mathrm{d} W_{s}=\sigma_{0}(\theta) \int_{0}^{\tau(a)} \frac{f\left(\tau^{-1}(t)\right)}{\sqrt{v_{0}\left(\tau^{-1}(t)\right)}} \mathrm{d} \hat{W}_{t} .
$$

Therefore,

$$
M_{\theta}=\sigma_{0}(\theta) \int_{0}^{1} \exp \left\{\theta^{H} F_{t}^{t}-\frac{\eta^{2}}{4}\left|\tau^{-1}(t)\right|^{2 H}\right\} \mathrm{d} \hat{B}_{t}
$$

where

$$
F_{u}^{t}=\eta \sqrt{\frac{H}{2}} \frac{\sigma_{0}(\theta)}{\theta^{H}} \int_{0}^{u} \frac{\left(\tau^{-1}(t)-\tau^{-1}(s)\right)^{H-1 / 2}}{\sqrt{v_{0}\left(\tau^{-1}(s)\right)}} \mathrm{d} \hat{W}_{s}, u \in[0, t] .
$$

Let

$$
G_{t}^{(k)}=H_{k}\left(F_{t}^{t},\left\langle F^{t}\right\rangle_{t}\right)
$$


Then, we have

$$
\begin{aligned}
M_{\theta} & =\sigma_{0}(\theta) \int_{0}^{1} \exp \left\{-\frac{\eta^{2}}{8}\left|\tau^{-1}(t)\right|^{2 H}\right\} \exp \left\{\theta^{H} F_{t}^{t}-\frac{\theta^{2 H}}{2}\left\langle F^{t}\right\rangle_{t}\right\} \mathrm{d} \hat{B}_{t} \\
& =\sigma_{0}(\theta) \int_{0}^{1} \exp \left\{-\frac{\eta^{2}}{8}\left|\tau^{-1}(t)\right|^{2 H}\right\} \sum_{k=0}^{\infty} G_{t}^{(k)} \frac{\theta^{H k}}{k !} \mathrm{d} \hat{B}_{t} .
\end{aligned}
$$

Lemma 5.1 We have (3) with

$$
\begin{aligned}
& M_{\theta}^{(0)}=\hat{B}_{1}, \\
& M_{\theta}^{(1)}=\int_{0}^{1} h_{\theta}(t) G_{t}^{(1)} \mathrm{d} \hat{B}_{t,} \\
& M_{\theta}^{(2)}=\int_{0}^{1}\left\{\frac{h_{\theta}(t)-1}{\theta^{2 H}}+h_{\theta}(t) \frac{G_{t}^{(2)}}{2}\right\} \mathrm{d} \hat{B}_{t}, \\
& M_{\theta}^{(3)}=2 \int_{0}^{1} F_{t}^{t} \mathrm{~d} t,
\end{aligned}
$$

where

$$
h_{\theta}(t)=\exp \left\{-\frac{\eta^{2}}{8}\left|\tau^{-1}(t)\right|^{2 H}\right\}
$$

Proof: For $M_{\theta}^{(i)}, i=0,1,2$, it suffices to show

$$
\left\|\int_{0}^{1} h_{\theta}(t) \sum_{k=J}^{\infty} G_{t}^{(k)} \frac{\theta^{H k}}{k !} \mathrm{d} \hat{B}_{t}\right\|_{2}=O\left(\theta^{H J}\right)
$$

for any $J \geq 3$. The proof for $M_{\theta}^{(3)}$ is similar and so omitted. It suffices to show

$$
E\left[\int_{0}^{1}\left|\sum_{k=J}^{\infty} G_{t}^{(k)} \frac{\theta^{H k}}{k !}\right|^{2} \mathrm{~d} t\right]=O\left(\theta^{2 H J}\right) .
$$

By the Cauchy-Schwarz inequality, the left hand side is dominated by

$$
\sum_{k=J}^{\infty} \theta^{H k} \sum_{k=J}^{\infty} \frac{\theta^{H k}}{(k !)^{2}} \int_{0}^{1} E\left[\left|G_{t}^{(k)}\right|^{2}\right] \mathrm{d} t
$$

Let

$$
G_{t, s}^{(k)}=H_{k}\left(F_{s}^{t},\left\langle F^{t}\right\rangle_{s}\right), \quad s \in[0, t]
$$


Then, by (12),

$$
\begin{aligned}
E\left[\left|G_{t}^{(k)}\right|^{2}\right] & =E\left[\left|G_{t, t}^{(k)}\right|^{2}\right] \\
& =k^{2} \int_{0}^{t} E\left[\left|G_{t, s}^{(k-1)}\right|^{2}\right] \mathrm{d}\left\langle F^{t}\right\rangle_{s} \\
& =k^{2}(k-1)^{2} \int_{0}^{t} \int_{0}^{s_{1}} E\left[\left|G_{t, s_{2}}^{(k-2)}\right|^{2}\right] \mathrm{d}\left\langle F^{t}\right\rangle_{s_{2}} \mathrm{~d}\left\langle F^{t}\right\rangle_{s_{1}} \\
& \leq(k !)^{2}\left\langle F^{t}\right\rangle_{t}^{k}=(k !)^{2}\left(\frac{\eta^{2}}{4} \frac{\left|\tau^{-1}(t)\right|^{2 H}}{\theta^{2 H}}\right)^{k} .
\end{aligned}
$$

Note that $\tau^{-1}(t) \leq \tau^{-1}(1)=\theta$. Therefore, for sufficiently small $\theta$,

$$
\sum_{k=J}^{\infty} \theta^{H k} \sum_{k=J}^{\infty} \frac{\theta^{H k}}{(k !)^{2}} \int_{0}^{1} E\left[\left|G_{t}^{(k)}\right|^{2}\right] \mathrm{d} t \leq\left(\frac{\eta^{2}}{4}\right)^{J} \frac{\theta^{2 H J}}{\left(1-\theta^{H}\right)\left(1-\theta^{H} \eta^{2} / 4\right)},
$$

which completes the proof.

Now we compute $a_{\theta}^{(i)}, b_{\theta}$ and $c_{\theta}$ based on Lemma 5.1. The following lemmas follow from the results in Section A by straightforward computations.

\section{Lemma 5.2}

$$
\begin{aligned}
& a_{\theta}^{(1)}(x)=-H_{3}(x) \phi(x) \rho \eta \sqrt{\frac{H}{2}} \frac{\sigma_{0}(\theta)}{\theta^{H}} \int_{0}^{1} h_{\theta}(t) \int_{0}^{t} \frac{\left(\tau^{-1}(t)-\tau^{-1}(s)\right)^{H-1 / 2}}{\sqrt{v_{0}\left(\tau^{-1}(s)\right)}} \mathrm{d} s \mathrm{~d} t \\
&=-H_{3}(x) \phi(x) \rho \eta \sqrt{\frac{H}{2}} \\
& \quad \times \frac{1}{\theta^{H} \sigma_{0}(\theta)^{3}} \int_{0}^{\theta} \exp \left\{-\frac{\eta^{2}}{8} t^{2 H}\right\} \int_{0}^{t}(t-s)^{H-1 / 2} \sqrt{v_{0}(s)} \mathrm{d} s v_{0}(t) \mathrm{d} t \\
& \sim-H_{3}(x) \phi(x) \frac{\rho \eta \sqrt{2 H}}{2(H+1 / 2)(H+3 / 2)} .
\end{aligned}
$$

\section{Lemma 5.3}

$$
\begin{aligned}
& a_{\theta}^{(2)}(x)=-H_{2}(x) \phi(x) \int_{0}^{1} \frac{h_{\theta}(t)-1}{\theta^{2 H}} \mathrm{~d} t \\
& -H_{4}(x) \phi(x) \rho^{2} \frac{\eta^{2} H}{4} \frac{\sigma_{0}(\theta)^{2}}{\theta^{2 H}} \int_{0}^{1} h_{\theta}(t)\left(\int_{0}^{t} \frac{\left(\tau^{-1}(t)-\tau^{-1}(s)\right)^{H-1 / 2}}{\sqrt{v_{0}\left(\tau^{-1}(s)\right)}} \mathrm{d} s\right)^{2} \mathrm{~d} t \\
& \sim H_{2}(x) \phi(x) \int_{0}^{1} \frac{\eta^{2}}{8 \theta^{2 H}}\left|\tau^{-1}(t)\right|^{2 H} \mathrm{~d} t-H_{4}(x) \phi(x) \rho^{2} \frac{\eta^{2} H}{(2 H+1)^{2}(2 H+2)}
\end{aligned}
$$

\section{Lemma 5.4}

$$
\begin{aligned}
a_{\theta}^{(3)}(x) & =-2 H_{2}(x) \phi(x) \rho \eta \sqrt{\frac{H}{2}} \frac{\sigma_{0}(\theta)}{\theta^{H}} \int_{0}^{1} \int_{0}^{t} \frac{\left(\tau^{-1}(t)-\tau^{-1}(s)\right)^{H-1 / 2}}{\sqrt{v_{0}\left(\tau^{-1}(s)\right)}} \mathrm{d} s \mathrm{~d} t \\
& \sim-2 H_{2}(x) \phi(x) \frac{\rho \eta \sqrt{2 H}}{2(H+1 / 2)(H+3 / 2)} .
\end{aligned}
$$




\section{Lemma 5.5}

$$
\begin{aligned}
c_{\theta}(x)= & H_{6}(x) \phi(x) \rho^{2} \frac{\eta^{2} H}{2} \frac{\sigma_{0}(\theta)^{2}}{\theta^{2 H}}\left(\int_{0}^{1} h_{\theta}(t) \int_{0}^{t} \frac{\left(\tau^{-1}(t)-\tau^{-1}(s)\right)^{H-1 / 2}}{\sqrt{v_{0}\left(\tau^{-1}(s)\right)}} \mathrm{d} s \mathrm{~d} t\right)^{2} \\
& +H_{4}(x) \phi(x) \rho^{2} \frac{\eta^{2} H}{2} \frac{\sigma_{0}(\theta)^{2}}{\theta^{2 H}} \int_{0}^{1} h_{\theta}(t)^{2}\left(\int_{0}^{t} \frac{\left(\tau^{-1}(t)-\tau^{-1}(s)\right)^{H-1 / 2}}{\sqrt{v_{0}\left(\tau^{-1}(s)\right)}} \mathrm{d} s\right)^{2} \mathrm{~d} t \\
& +H_{4}(x) \phi(x) \rho^{2} \eta^{2} H \frac{\sigma_{0}(\theta)^{2}}{\theta^{2 H}} \int_{0}^{1} h_{\theta}(t) \int_{0}^{t} \frac{\left(\tau^{-1}(t)-\tau^{-1}(s)\right)^{H-1 / 2}}{\sqrt{v_{0}\left(\tau^{-1}(s)\right)}} \mathrm{d} s \\
& +H_{4}(x) \phi(x) \frac{\eta^{2} H}{2} \frac{\sigma_{0}(\theta)^{2}}{\theta^{2 H}} \int_{0}^{1} h_{\theta}(t)^{2}\left(\int_{s}^{1} \frac{\left(\tau^{-1}(t)-\tau^{-1}(s)\right)^{H-1 / 2}}{\sqrt{v_{0}\left(\tau^{-1}(t)\right)}} \mathrm{d} t\right)^{2} \mathrm{~d} s \\
& +H_{2}(x) \phi(x) \frac{\eta^{2} H}{2} \frac{\sigma_{0}(\theta)^{2}}{\theta^{2 H}} \int_{0}^{1} h_{\theta}(t)^{2} \int_{0}^{t} \frac{\left(\tau^{-1}(t)-\tau^{-1}(s)\right)^{2 H-1}}{v_{0}\left(\tau^{-1}(s)\right)} \mathrm{d} \mathrm{d} t \\
\sim & H_{6}(x) \phi(x) \rho^{2} \frac{\eta^{2} H}{2(H+1 / 2)^{2}(H+3 / 2)^{2}}+H_{4}(x) \phi(x) \frac{2\left(1+\rho^{2}\right) \eta^{2} H}{(2 H+1)^{2}(2 H+2)} \\
& +H_{4}(x) \phi(x) \frac{\rho^{2} \eta^{2} H \beta(H+3 / 2, H+3 / 2)}{(H+1 / 2)^{2}} \\
& +H_{2}(x) \phi(x) \int_{0}^{1} \frac{\eta^{2}}{4 \theta^{2 H}}\left|\tau^{-1}(t)\right|^{2 H} \mathrm{~d} t .
\end{aligned}
$$

\section{A Conditional expectations of Wiener-Itô integrals}

Here we collect results on the conditional expectations of Wiener-Itô integrals that follow from Proposition 3 of Nualart et al [18]. Let $x \in \mathbb{R}$ and $B$ be a standard Brownian motion $\left(B_{0}=0\right)$. Let $f$ be a continuous function on

$$
\left\{(s, t) \in(0,1)^{2} ; s<t\right\}
$$

with

$$
\int_{0}^{1} \int_{0}^{t}|f(s, t)|^{2} \mathrm{~d} s \mathrm{~d} t<\infty
$$




\section{Lemma A.1}

$$
\begin{aligned}
E\left[\int_{0}^{1} \int_{0}^{t} f(s, t) \mathrm{d} B_{s} \mathrm{~d} t \mid B_{1}=x\right]= & H_{1}(x) \int_{0}^{1} \int_{0}^{t} f(s, t) \mathrm{d} s \mathrm{~d} t \\
E\left[\int_{0}^{1} \int_{0}^{t} f(s, t) \mathrm{d} B_{s} \mathrm{~d} B_{t} \mid B_{1}=x\right]= & H_{2}(x) \int_{0}^{1} \int_{0}^{t} f(s, t) \mathrm{d} s \mathrm{~d} t \\
E\left[\int_{0}^{1}\left(\int_{0}^{t} f(s, t) \mathrm{d} B_{s}\right)^{2} \mathrm{~d} B_{t} \mid B_{1}=x\right] & =H_{3}(x) \int_{0}^{1}\left(\int_{0}^{t} f(s, t) \mathrm{d} s\right)^{2} \mathrm{~d} t \\
& +H_{1}(x) \int_{0}^{1} \int_{0}^{t} f(s, t)^{2} \mathrm{~d} s \mathrm{~d} t \\
E\left[\int_{0}^{1}\left(\int_{s}^{1} f(s, t) \mathrm{d} B_{t}\right)^{2} \mathrm{~d} s \mid B_{1}=x\right]= & H_{2}(x) \int_{0}^{1}\left(\int_{s}^{1} f(s, t) \mathrm{d} t\right)^{2} \mathrm{~d} s \\
& +\int_{0}^{1} \int_{s}^{1} f(s, t)^{2} \mathrm{~d} t \mathrm{~d} s .
\end{aligned}
$$

\section{Lemma A.2}

$$
\begin{aligned}
& E\left[\left(\int_{0}^{1} \int_{0}^{t} f(s, t) \mathrm{d} B_{s} \mathrm{~d} B_{t}\right)^{2} \mid B_{1}=x\right]-\int_{0}^{1} \int_{0}^{t} f(s, t)^{2} \mathrm{~d} s \mathrm{~d} t \\
& =H_{4}(x)\left(\int_{0}^{1} \int_{0}^{t} f(s, t) \mathrm{d} s \mathrm{~d} t\right)^{2}+H_{2}(x) \int_{0}^{1}\left(\int_{0}^{t} f(s, t) \mathrm{d} s+\int_{t}^{1} f(t, u) \mathrm{d} u\right)^{2} \mathrm{~d} t .
\end{aligned}
$$

\section{References}

[1] Alòs, E., León, J.A. and Vives, J., On the short-time behavior of the implied volatility for jump-diffusion models with stochastic volatility, Finance Stoch. 11, (2007), 571-589.

[2] Alòs, E. and Shiraya, K.,Estimating the Hurst parameter from short term volatility swaps, to appear in Finance Stoch..

[3] Bayer, C., Friz, P.K. and Gatheral, J., Pricing under rough volatility, Quant. Finance 16:6, (2016), 887-904.

[4] Bayer, C. Friz, P.K., Gulisashvili, A., Horvath, B. and Stemper B., Shorttime near-the-money skew in rough fractional volatility models, to appear in Quant. Finance.

[5] Figueroa-López, J.E. and Ólafsson, S., Short-term asymptotics for the implied volatility skew under a stochastic volatility model with Lévy jumps, Finance Stoch. 20 (2016), 973-1020.

[6] Forde, M. and Zhang, H., Asymptotics for rough stochastic volatility models, SIAM J. Finan. Math., (2017), 8(1), 114-145. 
[7] Friz, P., Gerhold, S and Pinter, A., Option pricing in the moderate deviations regime, Math. Finance, (2018), 28(3), 962-988.

[8] Fukasawa, M., Asymptotic analysis for stochastic volatility: Martingale expansion, Finance Stoch. 15, (2011), 635-654.

[9] Fukasawa, M. The normalizing transformation of the implied volatility smile, Math. Finance 22, (2012) 753-762.

[10] Fukasawa, M., Short-time at-the-money skew and rough fractional volatility, Quant. Finance. 17:2, (2017), 189-198.

[11] Garnier, J. and Solna, K., Correction to Black-Scholes formula due to fractional stochastic volatility, SIAM J. Finan. Math., (2017), 8(1) 560-588.

[12] Gatheral, J., The Volatility Surface: A Practioner's Guide. (2006) (John Wiley \& Sons Inc: Hoboken, NJ).

[13] Gatheral, J., Jaisson, T. and Rosenbaum, M., Volatility is rough, Quant. Finance. 18:6, (2018), 933-949.

[14] Guennoun, H., Jacquier, A. and Roome, P., Asymptotic behaviour of the fractional Heston model, SIAM J. Finan. Math., (2018), 9(3), 1017-1045.

[15] Kunitomo, N. and Takahashi, A., On validity of the asymptotic expansion approach in contingent claim analysis, Ann. Appl. Probab., (2003), 13(3), 914-952.

[16] Medvedev, A. and Scaillet, O., A Simple Calibration Procedure of Stochastic Volatility Models with Jumps by Short Term Asymptotics, Research Paper No. 93, September 2003, FAME - International Center for Financial Asset Management and Engineering.

[17] Medvedev, A. and Scaillet, O., Approximation and calibration of shortterm implied volatilities under jump-diffusion stochastic volatility, The Review of Financial Studies , (2006), 20(2), 427-459.

[18] Nualart, D., Üstünel, A. S. and Zakai, M., On the moments of a multiple Wiener-Itô integral and the space induced by the polynomials of the integral. Stochastics 25 (1988), no. 4, 233-240.

[19] Osajima, Y., The asymptotic expansion formula of implied volatility for dynamic SABR model and FX hybrid model, 2006, UTMS 2006-29, The University of Tokyo.

[20] Pagliarani, S. and Pascucci, A., The exact Taylor formula of the implied volatility, Finance Stoch. 21 (2017), 661-718.

[21] Revuz, D. and Yor, M., Continuous Martingales and Brownian Motion, (1999), Springer-Verlag Berlin Heidelberg. 
[22] Yoshida, N., Asymptotic expansion for statistics related to small diffusions, J. Jpn. Stat. Soc., (1992) 22, 139-159.

[23] Yoshida, N., Malliavin calculus and martingale expansion, Bull. Sci. math. 125, 6-7 (2001) 431-456. 